University of Wollongong

Research Online

Australian Institute for Innovative Materials -

Papers

Australian Institute for Innovative Materials

$1-1-2017$

\title{
Photoexcited terahertz conductivity dynamics of graphene tuned by oxygen-adsorption
}

\author{
Zeyu Zhang \\ Shanghai University \\ Tie Lin \\ Chinese Academy Of Sciences \\ Xiao Xing \\ Shanghai University \\ Xian Lin \\ Shanghai University \\ Xiangjian Meng \\ Chinese Academy Of Sciences
}

See next page for additional authors

Follow this and additional works at: https://ro.uow.edu.au/aiimpapers

Part of the Engineering Commons, and the Physical Sciences and Mathematics Commons

Research Online is the open access institutional repository for the University of Wollongong. For further information contact the UOW Library: research-pubs@uow.edu.au 


\title{
Photoexcited terahertz conductivity dynamics of graphene tuned by oxygen- adsorption
}

\author{
Abstract \\ By using optical pump-terahertz $(\mathrm{THz})$ probe spectroscopy, the photoexcited terahertz conductivity \\ dynamics of chemical vapor deposition grown graphene is investigated in different atmospheric \\ environments. It is shown that the Fermi energy of doped graphene is engineered by oxygen adsorption \\ and desorption, which is probed by transient $\mathrm{THz}$ conductivity measurement. We show that the ultrafast \\ energy relaxation processes depend on Fermi energy (changed by environmental gas) and the density of \\ excited carriers (changed by photo-excitation fluence). The rise process of the negative conductivity \\ dynamics becomes less efficient upon decreasing the Fermi energy and/or increasing the pump fluence. \\ All findings show that the Fermi energy of graphene engineered by environmental gas allows us to tune \\ the ultrafast energy relaxation pathways in photoexcited graphene.

\section{Disciplines} \\ Engineering | Physical Sciences and Mathematics

\section{Publication Details} \\ Zhang, Z., Lin, T., Xing, X., Lin, X., Meng, X., Cheng, Z., Jin, Z. \& Ma, G. (2017). Photoexcited terahertz \\ conductivity dynamics of graphene tuned by oxygen-adsorption. Applied Physics Letters, 110 (11), \\ 111108-1-111108-5.

\section{Authors} \\ Zeyu Zhang, Tie Lin, Xiao Xing, Xian Lin, Xiangjian Meng, Zhenxiang Cheng, Zuanming Jin, and Guohong \\ $\mathrm{Ma}$
}




\section{Photoexcited terahertz conductivity dynamics of graphene tuned by oxygen-adsorption}

Zeyu Zhang, Tie Lin, Xiao Xing, Xian Lin, Xiangjian Meng, Zhenxiang Cheng, Zuanming Jin, and Guohong Ma

Citation: Appl. Phys. Lett. 110, 111108 (2017); doi: 10.1063/1.4978647

View online: http://dx.doi.org/10.1063/1.4978647

View Table of Contents: http://aip.scitation.org/toc/apl/110/11

Published by the American Institute of Physics

\section{Articles you may be interested in}

Strongly temperature dependent resistance of meander-patterned graphene

Appl. Phys. Lett. 110, 113104113104 (2017); 10.1063/1.4978597

Upscaling high-quality CVD graphene devices to 100 micron-scale and beyond

Appl. Phys. Lett. 110, 113502113502 (2017); 10.1063/1.4978643

Density of states evaluation of an insulating polymer by high-sensitivity ultraviolet photoemission spectroscopy Appl. Phys. Lett. 110, 111102111102 (2017); 10.1063/1.4978529

Polarization-independent electromagnetically induced transparency-like transmission in coupled guided-mode resonance structures

Appl. Phys. Lett. 110, 111106111106 (2017); 10.1063/1.4978670

Femtosecond-timescale buildup of electron mobility in GaAs observed via ultrabroadband transient terahertz spectroscopy

Appl. Phys. Lett. 110, 121102121102 (2017); 10.1063/1.4978648

Realization of direct bonding of single crystal diamond and Si substrates

Appl. Phys. Lett. 110, 111603111603 (2017); 10.1063/1.4978666
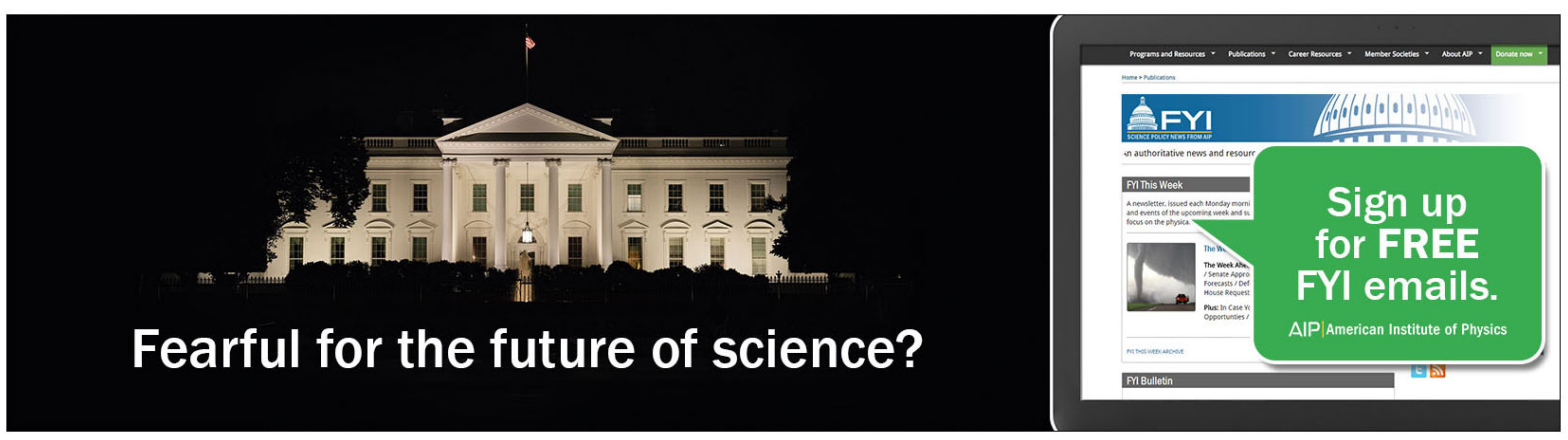


\title{
Photoexcited terahertz conductivity dynamics of graphene tuned by oxygen-adsorption
}

\author{
Zeyu Zhang, ${ }^{1}$ Tie Lin, ${ }^{2}$ Xiao Xing, ${ }^{1}$ Xian Lin, ${ }^{1}$ Xiangjian Meng, ${ }^{2}$ Zhenxiang Cheng, ${ }^{3}$ \\ Zuanming Jin, ${ }^{1, a)}$ and Guohong $\mathrm{Ma}^{1, a)}$ \\ ${ }^{1}$ Department of Physics, Shanghai University, Shanghai 200444, China \\ ${ }^{2}$ National Laboratory for Infrared Physics, Shanghai Institute of Technical Physics, \\ Chinese Academy of Sciences, Yu Tian Road 500, Shanghai 200083, People's Republic of China \\ ${ }^{3}$ Institute for Superconducting and Electronic Materials, University of Wollongong, Wollongong, \\ New South Wales 2522, Australia
}

(Received 24 November 2016; accepted 2 March 2017; published online 17 March 2017)

\begin{abstract}
By using optical pump-terahertz $(\mathrm{THz})$ probe spectroscopy, the photoexcited terahertz conductivity dynamics of chemical vapor deposition grown graphene is investigated in different atmospheric environments. It is shown that the Fermi energy of doped graphene is engineered by oxygen adsorption and desorption, which is probed by transient $\mathrm{THz}$ conductivity measurement. We show that the ultrafast energy relaxation processes depend on Fermi energy (changed by environmental gas) and the density of excited carriers (changed by photo-excitation fluence). The rise process of the negative conductivity dynamics becomes less efficient upon decreasing the Fermi energy and/ or increasing the pump fluence. All findings show that the Fermi energy of graphene engineered by environmental gas allows us to tune the ultrafast energy relaxation pathways in photoexcited graphene. Published by AIP Publishing. [http://dx.doi.org/10.1063/1.4978647]
\end{abstract}

Graphene has been considered as a promising material platform for high-speed electronics and optoelectronics owing to its high carrier mobility, broadband spectral response, and equilibration of photo-generated carriers. ${ }^{1,2}$ In recent years, graphene has been involved in terahertz (THz) technology. ${ }^{3-8} \mathrm{THz}$ photons can be used to investigate intraband transitions, ${ }^{9}$ which can be controlled by an electrode gate. ${ }^{10}$ In addition, the ultrafast $\mathrm{THz}$ photoconductivity dynamics of hot carriers in graphene are fundamentally important for the understanding of ultrafast energy relaxation pathways with carrier-carrier interactions and carrier-phonon emission processes. ${ }^{11}$

For an ideal intrinsic graphene, the $\mathrm{THz}$ photoconductivity is positive, which has been found with simple Drude models. George et al. observed an increase in terahertz pulse absorption for graphene grown epitaxially on a SiC substrate after interband optical excitation. ${ }^{12}$ The increase in absorption was attributed to an increase in the density of mobile charge carriers after photoexcitation. However, a comparable study on chemical vapor deposition (CVD)-grown graphene was found to demonstrate the opposite effect-photoinduced negative $\mathrm{THz}$ conductivity (photo-induced $\mathrm{THz}$ transparency). ${ }^{13}$ Indeed, the doping level has a critical impact on the photo-induced $\mathrm{THz}$ conductivity (including magnitude and sign) of graphene. ${ }^{14}$ For the heavily doped graphene, the negative photoconductivity has been previously attributed variously to stimulated $\mathrm{THz}$ emission, ${ }^{15}$ enhanced carrier scattering rate (momentum scattering time), ${ }^{13,16}$ and intraband carrier heating ${ }^{4,17}$ by several groups. Recently, the microscopic model ${ }^{11}$ clearly reveals that the transient carrier temperature, the Fermi level shifts, and the time- and momentum-dependent scattering rates are

\footnotetext{
${ }^{\text {a) }}$ Authors to whom correspondence should be addressed. Electronic addresses: physics_jzm@shu.edu.cn and ghma@staff.shu.edu.cn
}

all essential to capture the dynamic $\mathrm{THz}$ response completely. In addition, the energy relaxation of photoexcited carriers in graphene has been investigated extensively by varying the photon energies, the Fermi energy with gate voltages, and the pump fluencies. Previous studies reported that even a single molecule of a foreign substance can physically affect the electrical dc conductivity and optical response of graphene. ${ }^{18-20}$ However, the critical role of molecule-adsorption on the ultrafast energy relaxation pathways of graphene after photoexcitation remains unclear. ${ }^{21}$

In this letter, we utilized optical pump-THz probe spectroscopy to reveal the transient $\mathrm{THz}$ photoconductivity of CVD-grown graphene in different gaseous environments. Our findings show that upon decreasing the chemical potential, the carrier-carrier scattering efficiency decreases. In addition, the relaxation time of photo-induced $\mathrm{THz}$ conductivity increases with increasing pump fluence, which can be attributed to the hot phonon effect. The phenomenological thermodynamic model ${ }^{4,16}$ and the first-principle microscopic theory ${ }^{11}$ have been used to interpret all trends observed in our experiments.

The graphene in the present study is synthesized by a chemical vapor deposition (CVD) method under low pressure and high temperature. For an optical study, a poly methylmethacrylate PMMA layer is spin-coated on the as-grown graphene on $\mathrm{Cu}$-foil. Then, the $\mathrm{Cu}$-layer is etched out using a $0.1 \mathrm{M}$ ammonium per sulphate $\left(\mathrm{NH}_{4}\right)_{2} \mathrm{~S}_{2} \mathrm{O}_{8}$ solution. After rinsing with de-ionized water several times, graphene was transferred onto the fused silica substrate. Finally, the PMMA layer is dissolved in acetone three times. To remove any possible PMMA residual, the graphene sample is annealed in a vacuum at $350^{\circ} \mathrm{C}$ for $1.5 \mathrm{~h}$. Figure 1(a) shows the Raman spectra of our CVD-grown graphene on the fused silica substrate in air. The ratio of intensities of the 2D Raman peak $\left(2660 \mathrm{~cm}^{-1}\right)$ and $\mathrm{G}$ peak $\left(1583 \mathrm{~cm}^{-1}\right), \mathrm{I}_{2 \mathrm{D}} / \mathrm{I}_{\mathrm{G}} \sim 2$, is 


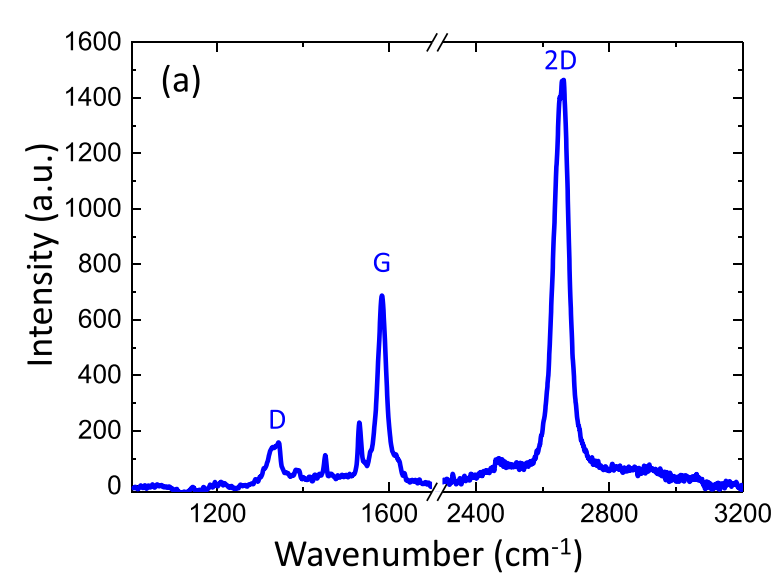

indicative of monolayer growth. The $\mathrm{G}$ peak center frequency can be used to estimate the Fermi energy by ${ }^{22}\left|\mathrm{E}_{\mathrm{F}}\right|=\left(\nu_{\mathrm{G}}-\right.$ $\left.1580 \mathrm{~cm}^{-1}\right) / 42 \mathrm{~cm}^{-1} \mathrm{eV}^{-1}$. We obtain the mean Fermi level of $\left|\mathrm{E}_{\mathrm{F}}\right|=71 \mathrm{meV}$ and then the average density of intrinsic dopant carriers by $\mathrm{N}_{\mathrm{i}}=\left(\mathrm{E}_{\mathrm{F}}\right)^{2} /\left(\hbar^{2} \mathrm{v}_{\mathrm{F}}^{2} \pi\right)$, where $\mathrm{v}_{\mathrm{F}}=1.1 \times 10^{6}$ $\mathrm{m} / \mathrm{s}$, and then $\mathrm{N}_{\mathrm{i}}=3.1 \times 10^{11} \mathrm{~cm}^{-2}$. The Fermi energy is negative owing to the known p-doping of our CVD graphene monolayer film.

To study the $\mathrm{THz}$ response of the CVD-grown graphene samples, we first measure the $\mathrm{THz}$ transmission through the substrate without graphene $\left(\mathrm{E}_{\mathrm{sub}}\right)$ and through the graphene on the substrate $\left(\mathrm{E}_{0}\right)$ without optical excitation. The attenuation of the terahertz waves comes for the conductivity of the intrinsic free carriers in graphene. By using the thin-film approximation (the Thinkham equation), we can obtain the intrinsic sheet complex conductivity by ${ }^{23,24}$

$$
\frac{E(\omega)}{E_{0}(\omega)}=\frac{n+1}{n+1+Z_{0} \hat{\sigma}(\omega)},
$$

where $\mathrm{n}=1.95$ is the refractive index of the fused silica substrate, and $\mathrm{Z}_{0}=377$ is the free space impedance. Figures 1(b) and 1(c) show the resulting complex sheet conductivity of the graphene samples in $\mathrm{N}_{2}$ and $\mathrm{O}_{2}$ within the $\mathrm{THz}$ range, respectively. The conductivity can be expressed in quantum units of $\mathrm{G}_{0}=2 \mathrm{e}^{2} / \mathrm{h}=7.73 \times 10^{-5} \mathrm{~S}$. Then, the real part of the sheet conductivity of the graphene sample in the DC limit is around $23.3 \mathrm{G}_{0}$ in $\mathrm{N}_{2}$ and $10.3 \mathrm{G}_{0}$ in $\mathrm{O}_{2}$. To understand the gas dependent transport properties, we fit the experimental conductivity by the standard Drude model, which is often employed for the transport of charge carriers in graphene ${ }^{13,25}$

$$
\hat{\sigma}(\omega)=\frac{D}{\pi} \frac{1}{(\Gamma-i \omega)},
$$

where $\Gamma$ is the average scattering rate for momentum changing collisions of charge carriers. The Drude weight $\mathrm{D}$ characterizes the magnitude of the response. $\mathrm{D}\left(\mathrm{N}_{2}\right)=7.22 \Omega^{-1} \cdot \mathrm{cm}^{-1}$ and $\mathrm{D}\left(\mathrm{O}_{2}\right)=29.63 \Omega^{-1} \cdot \mathrm{cm}^{-1}$ are obtained for the graphene sample in two gaseous environments. In addition, the carrier scattering times of $\tau\left(\mathrm{N}_{2}\right) \sim 26 \pm 2 \mathrm{fs}$ and $\tau\left(\mathrm{O}_{2}\right) \sim 3 \pm 1 \mathrm{fs}$ are simultaneously obtained by fitting. The number of carriers is calculated to be $|N|_{N_{2}} \approx 2.4 \times 10^{11} \mathrm{~cm}^{-2},|N|_{O_{2}} \approx 3.93$ $\times 10^{12} \mathrm{~cm}^{-2}$, respectively, by $\mathrm{D}=\left(v_{F} \frac{e^{2}}{h}\right)(\pi|N|)^{1 / 2}$, ${ }^{26}$ where $\mathrm{v}_{\mathrm{F}}=1.1 \times 10^{6} \mathrm{~ms}^{-1}$ is the Fermi velocity and $h$ is the Planck constant.

We now turn to the main focus of this paper, which is the effect of environmental gas on the carrier dynamics after photoexcitation. Ultrafast optical-pump THz-probe spectroscopy was performed using a $120 \mathrm{fs}, 1 \mathrm{kHz}$ Ti:sapphire regenerative amplifier system that excites the graphene samples with a photon energy of $1.55 \mathrm{eV}$. The $\mathrm{THz}$ pulses are generated by optical rectification and detected by electro-optic sampling in a pair of $1 \mathrm{~mm}$ thick, (110)-oriented ZnTe crystals. We measure the pump-induced changes of $\mathrm{THz}$ peak signals normalized to the $\mathrm{THz}$ transmission without photoexcitation $\left(\Delta \mathrm{T} / \mathrm{T}_{0}\right)$ for the same CVD graphene sample as a function of the pump-probe delay $\Delta \mathrm{t}$ under different atmospheric conditions. All measurements were performed at room temperature. As the monolayer graphene is thin, $\Delta \mathrm{T} / \mathrm{T}_{0}$ is related to the photoinduced sheet photoconductivity, i.e., $\Delta \sigma=\frac{1+n}{Z_{0}}\left(\frac{1}{1+\Delta T / T_{0}}-1\right) \cdot{ }^{24}$ Therefore, the observed positive $\Delta \mathrm{T} / \mathrm{T}_{0}$ indicates a suppression of the photoconductivity in the graphene sample.

Figure 2(a) shows the negative photoinduced $\mathrm{THz}$ conductivities of our CVD graphene sample as a function of the pump-probe delay time for various gaseous atmospheres, with an optical pump fluence fixed at $50 \mu \mathrm{J} / \mathrm{cm}^{2}$. We find the dynamical traces that show a subpicosecond rise followed by a several picoseconds mono-exponential decay. The first rise in the THz conductivities has been ascribed to three main processes: ${ }^{4,16}$ the creation of initial electron-hole pairs and subsequent ultrafast energy relaxes through two relaxation channels: (1) The carrier-carrier scattering (between the photoexcited carriers and the carriers in the Fermi level) and (2) the optical phonon emission transfers energy from the hot carrier to the lattice. It is important to note that the ultrafast energy relaxation takes place during the first hundreds of femtoseconds after photoexcitation. We observe that the THz conductivity dynamics are dramatically different for the same CVD graphene sample exposed to different gaseous atmospheres. Not only the peak value but also the dynamical response of the $\mathrm{THz}$ conductivity is strongly dependent on the gas molecules adsorbed. To quantify these results, we employ a phenomenological model to fit the time-resolved photoconductivity, in which a rise component with an effective time 


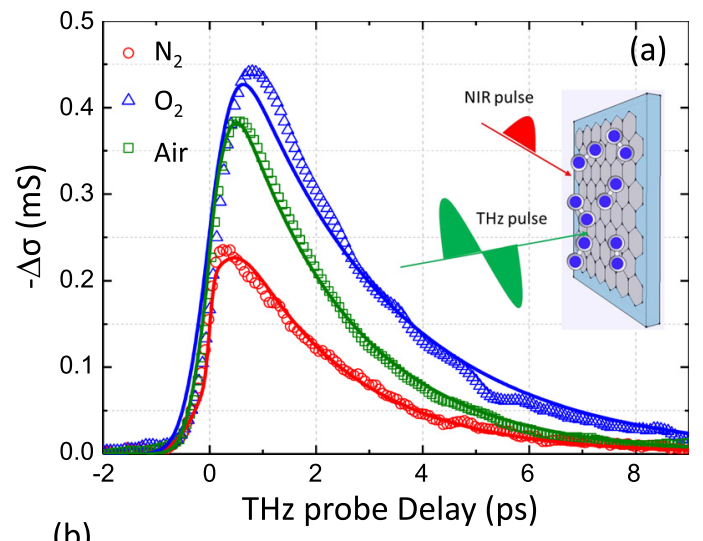

(b)

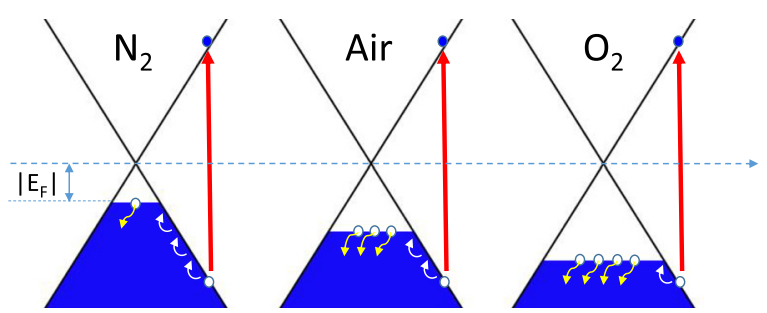

FIG. 2. (a) $-\Delta \sigma$ as a function of optical pump-THz probe delay probed for different dry gaseous atmospheres $\left(\mathrm{N}_{2}\right.$, air, and $\left.\mathrm{O}_{2}\right)$ at room temperature. Solid curves are the fits with an effective rise time constant and an exponential decay time constant (see text). The inset shows the schematic of the monolayer graphene exposed to molecule-adsorption along with the optical pump and THz probe measurement. (b) Typical Dirac-Fermion bands in graphene in three different gas environments. The doping level of CVD grown graphene is modulated by adsorption of oxygen. The schematics also show the ultrafast energy relaxation of the photoexcited carriers and the multiple hot electron excitation in the three different Fermi levels.

constant $\tau_{\text {rise }}$ and a mono-exponential decay with a time constant $\tau_{\text {decay }}$ are given by ${ }^{16}$

$$
\begin{aligned}
-\Delta \sigma & =A \times \operatorname{Conv}\left(e^{-t / \tau_{\text {decay }}}, \tau_{\text {pulse }}\right) \\
& +B \times \operatorname{Conv}\left(e^{-t / \tau_{\text {decay }}}, \tau_{\text {slow rise }}\right),
\end{aligned}
$$

where $\operatorname{Conv}\left(e^{-t / \tau_{\text {decay }}}, \tau\right)$ means the convolution of $e^{-t / \tau_{\text {decay }}}$ with a Gaussian pulse with the pulse width of $\tau$. The effective rise time is the amplitude-weight average of two: the laser pulse duration of $\tau_{\text {pulse }}=120$ fs and a variable relative slow rise component $\left(\tau_{\text {slow rise }}\right)$. The effective rise time constant $\tau_{\text {rise }}=(\mathrm{A}+\mathrm{B}) /\left[\left(\mathrm{A} / \tau_{\text {pulse }}\right)+\left(\mathrm{B} / \tau_{\text {slow rise }}\right)\right],{ }^{16}$ which indicates how fast the initial photoexcited energy relaxation. In our case, as shown in Fig. 2(a), with the increasing amount of adsorbed oxygen, $\tau_{\text {rise }}$ increases from $0.5 \pm 0.02$ ps (in $\mathrm{O}_{2}$ ) and $0.42 \pm 0.02 \mathrm{ps}$ (in air) to $1.0 \pm 0.04 \mathrm{ps}$ (in $\mathrm{N}_{2}$ ).

We indeed note that the $\mathrm{THz}$ conductivity signals of graphene exposed to gases can be "reset" replacing another dry gas, which indicates that the gas molecules are physisorbed (reversibly dopes graphene) rather than chemisorbed to the graphene surface (irreversibly opens the gap of graphene). ${ }^{18,21}$ The adsorption of molecules can add or reduce the physically adsorbed dopants of graphene locally and then shift its Fermi energy level, hence altering the density of intrinsic carriers. ${ }^{19}$ As previously reported by Ryu et al., the upshift of the Raman $G$ band represents positive charge doping. ${ }^{18}$

The slowing down of the effective rise time constant in $\mathrm{N}_{2}$ gas can be explained with the energy relaxation through intraband carrier-carrier scattering. As shown in Fig. 2(b), in a phenomenological picture, the slowdown of $\tau_{\text {rise }}$ means that larger numbers of carrier scattering events are required for the photoexcited carriers to perform their energy relaxation cascade and therefore for the Fermi level are shifted further close to the Dirac point under environmental $\mathrm{N}_{2}$ gas, thus increasing the $\mathrm{E}_{0} / \mathrm{E}_{\mathrm{F}}{ }^{7,16}$ Our measurements based on $\mathrm{THz}$ data are consistent with the Raman measurement, ${ }^{18}$ which reveals that the $\left|\mathrm{E}_{\mathrm{F}}\right|$ of graphene in $\mathrm{O}_{2}$ is larger than that in $\mathrm{N}_{2}$, as shown in Fig. 2(b).

Figure 3(a) shows the $\mathrm{THz}$ conductivity dynamics for increased pump fluencies with fixed Fermi levels for the $\mathrm{N}_{2}$, $\mathrm{O}_{2}$, and air exposed graphene, respectively. Figure 3(b) represents $\tau_{\text {rise }}$ as a function of pump fluence in $\mathrm{N}_{2}$, air, and $\mathrm{O}_{2}$, respectively. Our results indicate that an increase in the pump fluence leads to a slight further slower rise dynamics of $\mathrm{THz}$ conductivity in all cases. Figure 4(a) shows that the peak value $-\Delta \sigma$ increases with an apparent saturation behavior in the photo-excited conductivity with increasing pump fluence. In addition, we find that $-\Delta \sigma$ in $\mathrm{N}_{2}$ is smaller than that in $\mathrm{O}_{2}$ at each pump fluence, as shown in Figs. 4(a) and 2(a).

A phenomenological thermodynamics model can be directly used to understand (1) that the negative photoconductivity in $\mathrm{O}_{2}$ is larger than that in $\mathrm{N}_{2}$ and air and (2) the dependence of the extracted effective rise times on the Fermi energy and pump fluence. Predicted by the thermodynamic model, ${ }^{16,27,28}$ the magnitude of $-\Delta \sigma_{\text {peak }}$ increases as the Fermi level gets further away from the Dirac point (i.e., increasing the density of intrinsic carriers and the heating efficiency). This is in line with our observations that larger negative photoconductivity is observed in $\mathrm{O}_{2}$ at fixed pump fluence. Upon photoexcitation, to keep the total number of conduction band carriers constant, the photo-induced increase in carrier temperature will lead to a decrease in the chemical potential $\left(\left|\mathrm{E}_{\mathrm{F}}\right|\right)$. For the case of lower $\left|\mathrm{E}_{\mathrm{F}}\right|$, the heating efficiency will decrease owing to the slower intraband carrier-carrier scattering, ${ }^{16}$ as shown schematically in Fig. 2(b). Notably, our experimental results are also in line with the microscopic theory; the reduced efficiency of carriercarrier scattering scales with the decreasing carrier density at the Fermi level. ${ }^{11}$ This is the reason for the further slowing of the rise dynamics occurring at the lower $\left|E_{F}\right|$ in $N_{2}$ than in $\mathrm{O}_{2}$ with fixed pump fluence and at higher pump fluence in each atmosphere of $\mathrm{N}_{2}$, air, and $\mathrm{O}_{2}$. Such rise dynamics are consistent with the rise times in Ref. 16.

As shown in Fig. 4(a), we observed that the peak value $\left|\Delta \sigma_{\text {peak }}\right|$ semi-qualitatively scales as pump fluences $\mathrm{F}^{0.11 \pm 0.02}$, $\mathrm{F}^{0.15 \pm 0.05}$, and $\mathrm{F}^{0.24 \pm 0.05}$ for the graphene sample in air, $\mathrm{O}_{2}$, and $\mathrm{N}_{2}$, respectively. The sub-linearity is related to the sublinear dependence of the $\mathrm{THz}$ photoconductivity on electron temperature and chemical potential and is also connected to the interaction between the efficiency of carrier heating and the energy relaxation by optical-phonon emission. These two relaxation channels are reinforcing one another, as explained in the microscopic model. ${ }^{11}$

Finally, let us discuss the relaxation time of the photoexcited conductivity in our CVD-grown graphene with relatively high doping density. The decay of photoconductivity 

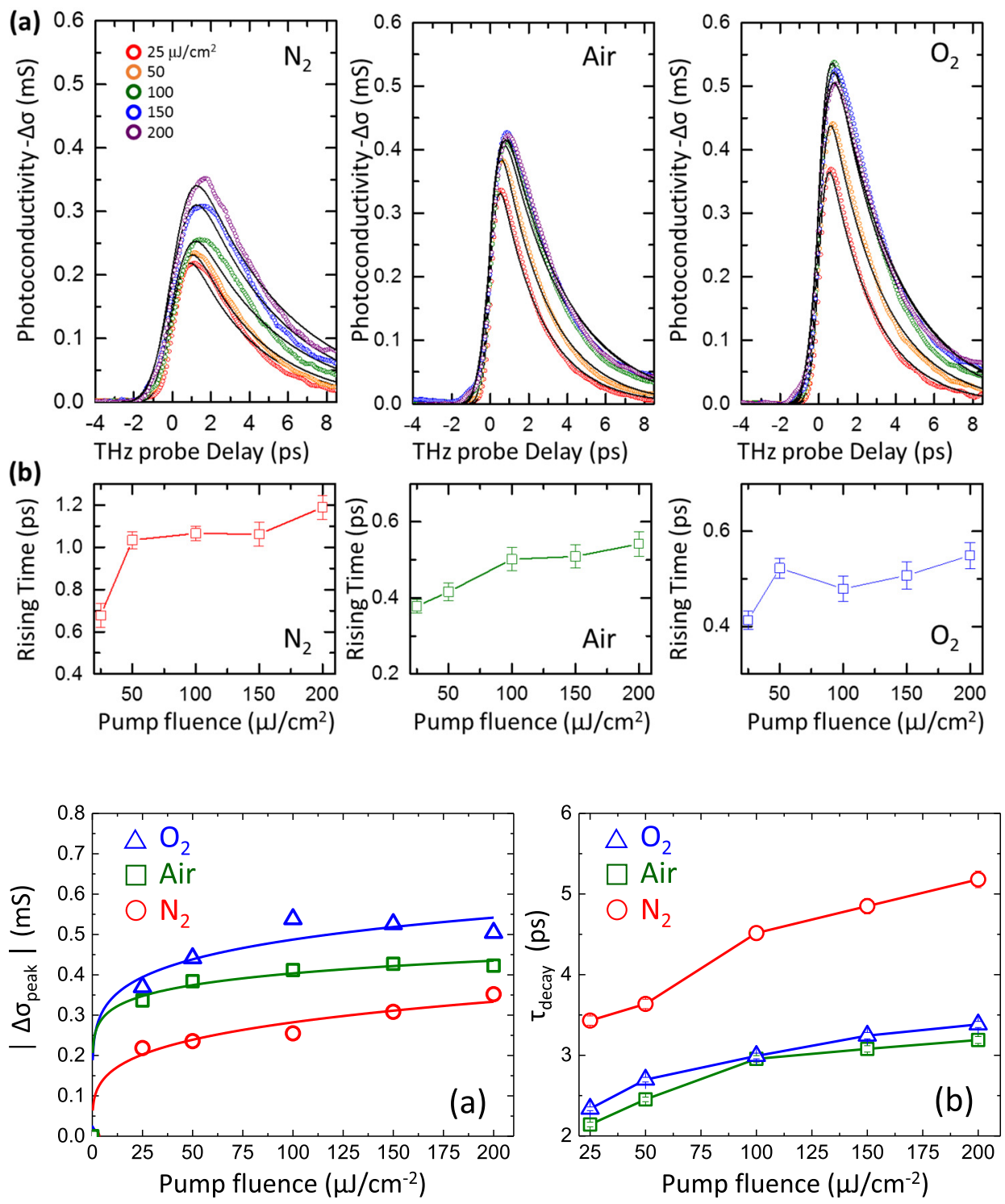

FIG. 3. (a) Transient photoexcited THz negative conductivity for different pump fluences in $\mathrm{N}_{2}$, air, and $\mathrm{O}_{2}$. Solid curves are the fits with a phenomenological model. (b) The extracted values for $\tau_{\text {rise }}$ as a function of pump fluence in $\mathrm{N}_{2}$, air, and $\mathrm{O}_{2}$ at room temperature.
FIG. 4. (a) Peak value of the photoexcited negative $\mathrm{THz}$ conductivity $\left(\left|\Delta \sigma_{\text {peak }}\right|\right)$ extracted from Fig. 3(a) as a function of pump fluence. (b) Carrier relaxation times extracted from fits as a function of pump fluence in dry $\mathrm{N}_{2}$, air, and $\mathrm{O}_{2}$. follows closely a mono-exponential relaxation under all experimental conditions. This relaxation time has been previously ascribed to the direct coupling between electrons and acoustic phonons in the presence of lattice defects. ${ }^{29,30}$ More recently, the $\mathrm{THz}$ carrier dynamics can be quantitatively explained using a microscopic density-matrix theory. ${ }^{11}$ Based on the microscopic model, the carrier-acoustic-phonon scattering has been found not to be important on the picosecond timescales. The mono-exponential relaxation is fully accounted for by the combined effect of carrier-carrier and carrier-optical-phonon scattering in the absence of disorder. Figure 4(b) shows the summary of extracted carrier relaxation times as a function of pump fluence. We note that the relaxation times of the doped graphene samples exposed to any gases slightly increase with increasing pump fluence. The magnitudes of $\tau_{\text {decay }}$ are very similar in the range of $\sim 2-3.5 \mathrm{ps}$ for the graphene sample in air and $\mathrm{O}_{2}$. The magnitudes of $\tau_{\text {decay }}$ are consistent with the relaxation times in Ref. 11. The magnitude of $\tau_{\text {decay }}$ for the relative lightly doped graphene sample in $\mathrm{N}_{2}$ is longer than that for highly doped graphene in air and $\mathrm{O}_{2}$. This is due to the lower $\left|\mathrm{E}_{\mathrm{F}}\right|$ for the graphene sample in $\mathrm{N}_{2}$, the less efficient optical phonon emission becomes, because fewer carriers can emit an optical phonon due to the reduced phase space close to the Dirac point. It is noted that the slight increase in the relaxation times with increasing pump fluence has also been experimentally observed by Mihnev et al. ${ }^{11}$ Within the microscopic theory, the longer relaxation time observed at high pump fluence is due to the hot phonon effects. ${ }^{11}$ Certainly, we would emphasis that, from the experimental point of view, a validation of the full relaxation dynamics would require temperature- and photo-energy-dependent measurements. From the theoretical point of view, ${ }^{31,32}$ to exactly capture the $\mathrm{THz}$ conductivity dynamics, all the transient carrier temperature and transient Fermi level shifting under photo-excitation and time- and momentum-dependent scattering behaviors are required, ${ }^{11}$ which are beyond the scope of this paper.

In conclusion, ultrafast time-resolved $\mathrm{THz}$ spectroscopy has been utilized to study the photo-induced hot-carrier generation and relaxation dynamics of environmental gas exposed CVD graphene. Our findings show that decreasing oxygen adsorption has an impact on decreasing $\left|\mathrm{E}_{\mathrm{F}}\right|$ and then tune the negative $\mathrm{THz}$ conductivity of gas-adsorbed CVD graphene. Both the phenomenological thermodynamics 
model and the microscopic theory capture all trends observed in our experiments. The results in the end led us to our main finding that the atmospheric gas can be used as a degree of freedom to control the ultrafast energy relaxation pathway. In other words, the gas environmental influence should be taken into consideration for the graphene-based device designs and applications.

This work was supported by the National Natural Science Foundation of China (NSFC, Grant Nos. 11674213 and 11604202) and the Research Innovation Fund of the Shanghai Education Committee (Grant No. 14ZZ101). Z.J. thanks the Young Eastern Scholar (Grant No. QD2015020) at Shanghai Institutions of Higher Learning and the Colleges and Universities Young Teachers Training Funding Program of Shanghai Municipal Education Commission (Grant No. ZZSD15098).

${ }^{1}$ F. Bonaccorso, Z. Sun, T. Hasan, and A. C. Ferrari, Nat. Photonics 4, 611 (2010).

${ }^{2}$ Q. Bao and K. P. Loh, ACS Nano 6, 3677 (2012).

${ }^{3}$ S. Winnerl, M. Orlita, P. Plochocka, P. Kossacki, M. Potemski, T. Winzer, E. Malic, A. Knorr, M. Sprinkle, C. Berger, W. A. de Heer, H. Schneider, and M. Helm, Phys. Rev. Lett. 107, 237401 (2011).

${ }^{4}$ K. J. Tielrooij, J. C. W. Song, S. A. Jensen, A. Centeno, A. Pesquera, A. Zurutuza Elorza, M. Bonn, L. S. Levitov, and F. H. L. Koppens, Nat. Phys. 9, 248 (2013).

${ }^{5}$ H. Choi, F. Borondics, D. A. Siegel, S. Y. Zhou, M. C. Martin, A. Lanzara, and R. A. Kaindl, Appl. Phys. Lett. 94, 172102 (2009).

${ }^{6}$ J. M. Dawlaty, S. Shivaraman, M. Chandrashekhar, F. Rana, and M. G. Spencer, Appl. Phys. Lett. 92, 042116 (2008).

${ }^{7}$ J. C. W. Song, K. J. Tielrooij, F. H. L. Koppens, and L. S. Levitov, Phys. Rev. B 87, 155429 (2013).

${ }^{8}$ J. H. Strait, H. Wang, S. Shivaraman, V. Shields, M. Spencer, and F. Rana, Nano Lett. 11, 4902 (2011).

${ }^{9}$ L. Prechtel, L. Song, D. Schuh, P. Ajayan, W. Wegscheider, and A. W. Holleitner, Nat. Commun. 3, 646 (2012).

${ }^{10}$ Y. Sato, K. Takai, and T. Enoki, Nano Lett. 11, 3468 (2011).
${ }^{11}$ M. T. Mihnev, F. Kadi, C. J. Divin, T. Winzer, S. Lee, C.-H. Liu, Z. Zhong, C. Berger, W. A. de Heer, E. Malic, A. Knorr, and T. B. Norris, Nat. Commun. 7, 11617 (2016).

${ }^{12}$ P. A. George, J. Strait, J. Dawlaty, S. Shivaraman, M. Chandrashekhar, F. Rana, and M. G. Spencer, Nano Lett. 8, 4248 (2008).

${ }^{13}$ G. Jnawali, Y. Rao, H. Yan, and T. F. Heinz, Nano Lett. 13, 524 (2013).

${ }^{14}$ J. Kim, J. Oh, C. In, Y. S. Lee, T. B. Norris, S. C. Jun, and H. Choi, ACS Nano 8, 2486 (2014).

${ }^{15}$ S. Boubanga-Tombet, S. Chan, T. Watanabe, A. Satou, V. Ryzhii, and T. Otsuji, Phys. Rev. B 85, 035443 (2012).

${ }^{16}$ S. A. Jensen, Z. Mics, I. Ivanov, H. S. Varol, D. Turchinovich, F. H. L. Koppens, M. Bonn, and K. J. Tielrooij, Nano Lett. 14, 5839 (2014).

${ }^{17}$ L. Malard, K. Mak, A. Neto, N. Peres, and T. Heinz, New J. Phys. 15, 015009 (2013).

${ }^{18}$ S. Ryu, L. Liu, S. Berciaud, Y. Yu, H. Liu, P. Kim, G. W. Flynn, and L. E. Brus, Nano Lett. 10, 4944 (2010).

${ }^{19}$ B. K. Daas, W. K. Nomani, K. M. Daniels, T. S. Sudarshan, G. Koley, and M. V. S. Chandrashekhar, Mater. Sci. Forum 717, 665 (2012).

${ }^{20}$ Y. Sano, I. Kawayama, M. Tabata, K. A. Salek, H. Murakami, M. Wang, R. Vajtai, P. M. Ajayan, J. Kono, and M. Tonouchi, Sci. Rep. 4, 6046 (2014).

${ }^{21}$ C. J. Docherty, C.-T. Lin, H. J. Joyce, R. J. Nicholas, L. M. Herz, L.-J. Li, and M. B. Johnston, Nat. Commun. 3, 1228 (2012).

${ }^{22}$ A. Das, S. Pisana, B. Chakraborty, S. Piscanec, S. K. Saha, U. V. Waghmare, K. S. Novoselov, H. R. Krishnamurthy, A. K. Geim, A. C. Ferrari, and A. K. Sood, Nat. Nanotechnol. 3, 210 (2008).

${ }^{23}$ S. A. Jensen, R. Ulbricht, A. Narita, X. Feng, K. Müllen, T. Hertel, D. Turchinovich, and M. Bonn, Nano Lett. 13, 5925 (2013).

${ }^{24}$ H. Yan, B. An, Z. Fan, X. Zhu, X. Lin, Z. Jin, and G. Ma, Appl. Phys. A 122, 414 (2016).

${ }^{25}$ Q. Li, Z. Tian, X. Zhang, R. Singh, L. Du, J. Gu, J. Han, and W. Zhang, Nat. Commun. 6, 7082 (2015).

${ }^{26}$ S. Kar, D. R. Mohapatra, E. Freysz, and A. K. Sood, Phys. Rev. B 90, 165420 (2014).

${ }^{27}$ I. Ivanov, M. Bonn, Z. Mics, and D. Turchinovich, Eur. Phys. Lett. 111, 67001 (2015).

${ }^{28}$ Z. Mics, K.-J. Tielrooij, K. Parvez, S. A. Jensen, I. Ivanov, X. Feng, K. Müllen, M. Bonn, and D. Turchinovich, Nat. Commun. 6, 7655 (2015).

${ }^{29}$ J. C. W. Song, M. Y. Reizer, and L. S. Levitov, Phys. Rev. Lett. 109, 106602 (2012).

${ }^{30}$ M. W. Graham, S.-F. Shi, D. C. Ralph, J. Park, and P. L. McEuen, Nat. Phys. 9, 103 (2013).

${ }^{31}$ R. Bistritzer and A. H. MacDonald, Phys. Rev. Lett. 102, 206410 (2009).

${ }^{32}$ W.-K. Tse and S. Das Sarma, Phys. Rev. B 79, 235406 (2009). 